\title{
Crack propagation in a toughened epoxy adhesive under repeated impacts
}

\author{
Ian A. Ashcroft ${ }^{\mathrm{a}, *}$, Vadim V. Silberschmidt ${ }^{\mathrm{a}}$, Benjamin Echard ${ }^{\mathrm{a}}$ and Juan Pablo Casas Rodriguez ${ }^{\mathrm{b}}$ \\ ${ }^{a}$ Wolfson School of Mechanical and Manufacturing Engineering, Loughborough University, Leicestershire, LE11 \\ $3 T U, U K$ \\ ${ }^{\mathrm{b}}$ Ingenieria Mecanica, Universidad de los Andes, Bogota, Colombia
}

Received 15 Feburary 2010

Revised 10 August 2010

\begin{abstract}
Adhesives are being increasingly used in structural applications, especially in aerospace, automotive and naval structures, making their structural integrity an important issue. In-service loading histories of such structures usually contain low-energy impacts, repetition of which can significantly affect their performance. This paper deals with the behaviour of the toughened epoxy adhesive FM73 under repeated impacts, known as impact fatigue. Izod impact fatigue tests were performed on FM73 specimens in order to study the evolution of damage and to characterise this via measurable parameters, such as the maximum force and the contact time. A finite element model was developed to simulate the impact tests and this was used to calculate the dynamic strain energy release rate, which was compared with that determined using a simple analytical method. A relationship between the maximum dynamic strain energy release rate and impact fatigue crack growth rate was established that was used as the basis of an impact fatigue crack growth law.
\end{abstract}

Keywords: Dynamic strain energy release rate, impact fatigue, crack propagation, adhesive, fracture mechanics, Izod test

\section{Introduction}

Engineering adhesives are now widely used in structural applications, especially in the aerospace industry, as an alternative to rivets and other mechanical joints. In these applications, important advantages of adhesive bonding include weight reduction, sealing ability, increased stiffness, the ability to join thin sections and the removal of the requirement to create a hole in the parent material. Of course, there are also certain disadvantages with adhesive bonding, such as sensitivity to manufacturing conditions and the in-service environment. However, most of the perceived problems are surmountable and, hence, an increase in structural applications for adhesive bonding has been seen in recent years. In order to use adhesive bonding safely and efficiently in structural applications, an ability to predict the response of the adhesive joint to the in-service environment and loading is required. In order to achieve this, knowledge of the response of the bonded joint to all the loading situations experienced is required, together with the development of procedures for predicting this behaviour that can be used by the designer. There has been much progress in this regard in recent years, e.g. see Oechsner and da Silva [10], however, impact fatigue is still a relatively little studied mode of loading for bonded joints.

Adhesively bonded joints in most structural applications, such as aerospace structures, are exposed to complex inservice loading histories, containing cycles with various amplitudes and durations. Generally, such cyclic loading is analysed in terms of traditional fatigue tests, which employ mainly a harmonic type of external excitation. However, some of the real-life loading cycles are impacts and are poorly represented by sinusoidal loading. Repetition of

*Corresponding author. Tel.: +44 1509 227535; Fax: +44 1509 227502; E-mail: I.A.Ashcroft@lboro.ac.uk. 


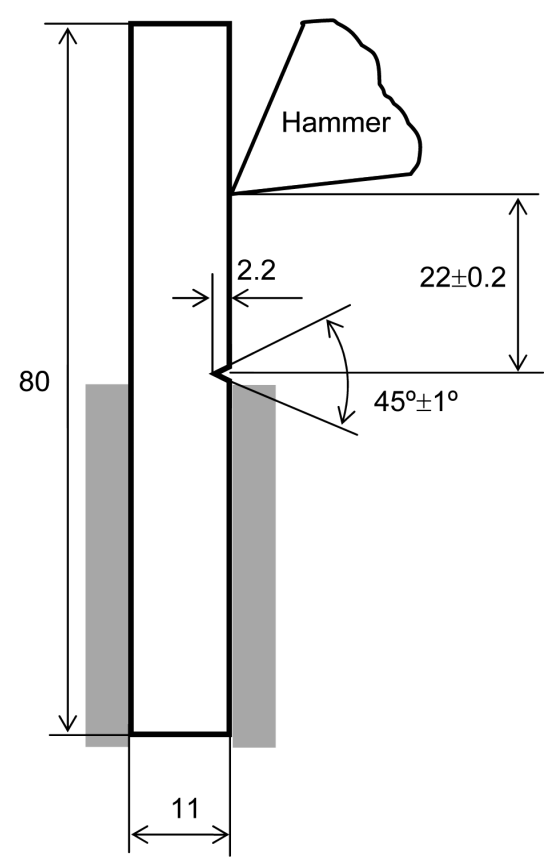

Fig. 1. Dimensions of Izod impact test samples.

such impact events - known as impact fatigue - has been shown to have a significantly more detrimental effect on the structural integrity and reliability of adhesively-bonded joints than sinusoidal loading [4-6]. The rate of crack growth in bonded joints under conditions of impact fatigue were found to be up to two orders of magnitude higher than in conventional, non-impact, fatigue.

The experimental study of bonded joints is complicated by the effects of adherends and their interfaces with the adhesive; this results in multi-mechanism fracture scenarios [7] that can include deviations of a propagating crack into a composite adherend [3]. To remove this complication, this paper considers fracture evolution in bulk specimens of adhesive tested under repeated impacts, giving an opportunity to study the behaviour of a typical aerospace adhesive unaffected by the adherends and interfaces. This paper consists of an experimental part, in which the results from impact fatigue testing notched bulk samples of the adhesive in an Izod test configuration are presented, and an analytical one, in which an impact fatigue crack growth law for the adhesive is determined.

\section{Experimental procedure}

For the experimental tests, a toughened epoxy film adhesive widely used in the aerospace industry adhesive, Cytec FM-73, was used. Plates of FM73 were manufactured according to the Cytec technical datasheet. Sheets of the uncured adhesive were cut to fit in a $100 \mathrm{~mm} \times 100 \mathrm{~mm} \times 4 \mathrm{~mm}$ mould. Layers of the films, each $0.18 \mathrm{~mm}$ thick, were assembled to obtain a sheet thickness of approximately $4 \mathrm{~mm}$. Curing was carried out under pressure, by heating for 30 minutes up to $120^{\circ} \mathrm{C}$ and then holding at this temperature for a further 60 minutes. The cured plates were then laser cut to the required specimen shape, which is shown in Fig. 1 . The $45^{\circ}$ angle notches were made using a milling machine.

A CEAST RESIL impact testing machine was used for the Izod impact tests. This is a pendulum hammer machine, with a pneumatic brake and automatic hammer positioning. The machine is software controlled, allowing the energy applied to the specimen, the number of impacts in a series, the filter frequency, the number of data points captured and the hammer type to be specified. The system provides a high time resolution force signal, with up to several thousand data points for each impact, lasting 6-10 ms in our case.

Before each impact, the hammer is raised to a specific height corresponding to the chosen initial potential energy. Then, the hammer is released to hit the specimen. A piezo-electric sensor placed in the machine's anvil is used to 


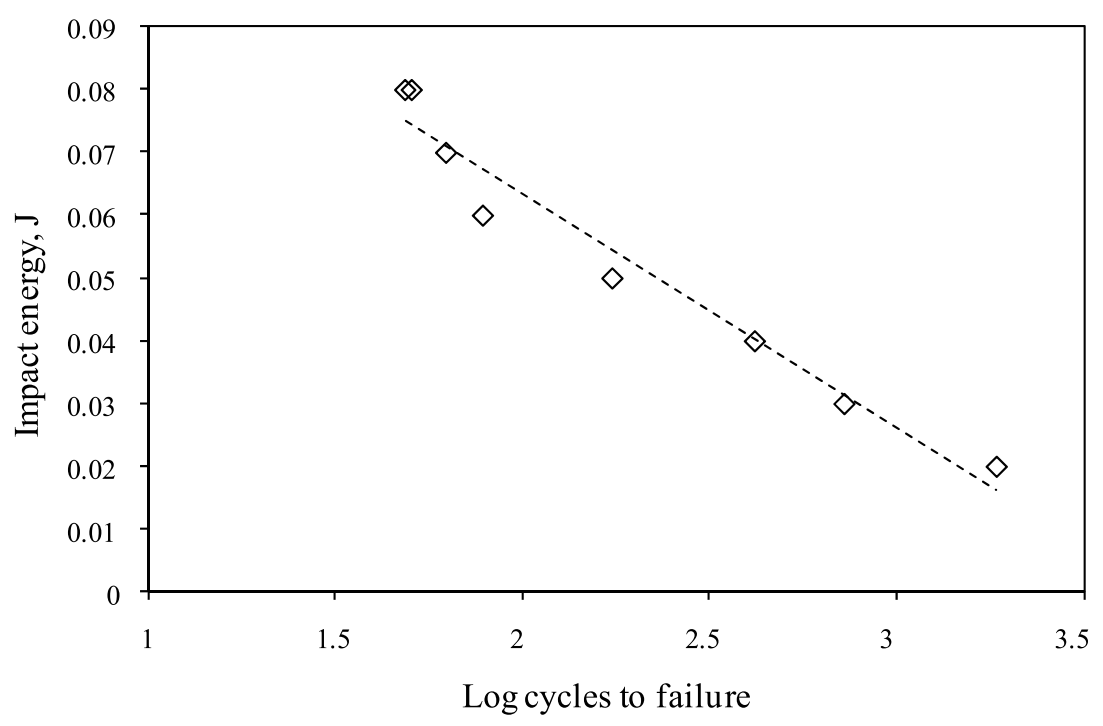

Fig. 2. Plot of impact fatigue life as a function of impact energy.

measure the reaction force, $F$, as a function of time, $t$, for each impact. The velocity, $V$, deformation, $d$, and energy, $U$, can be calculated from the force and time data, as shown below.

$$
V_{i}=V_{i-1}-\Delta t_{i-1, i}\left(\frac{\langle F\rangle_{i-1, i}}{m}-g\right),
$$

where $\langle F\rangle_{i-1, i}=\frac{1}{2}\left(F_{i-1}+F_{i}\right), g$ is the gravitational acceleration and $\Delta t_{i-1, i}=t_{i}-t_{i-1}$,

$$
d_{i}=d_{i-1}-\Delta t_{i-1, i}\langle V\rangle_{i-1, i},
$$

where $\langle V\rangle_{i-1, i}=\frac{1}{2}\left(V_{i-1}+V_{i}\right)$,

$$
U_{i}=U_{i-1}-\Delta t_{i-1, i}\langle F V\rangle_{i-1, i}
$$

where $\langle F V\rangle_{i-1, i}=\frac{1}{2}\left((F V)_{i-1}+(F V)_{i}\right)$.

Single-impact and impact-fatigue tests can be performed with this machine. In this work single-impact tests were carried out to determine the critical strain energy release rate for impact conditions. Impact-fatigue tests were then conducted to study the behaviour of the adhesive under repeated impacts. The specimen dimensions used, which are defined by ISO-180 (1997), were the same in both types of tests and in both cases fatigue tests were carried out over a range of impact energies. In order to observe crack propagation in the material, every 5, 10 or 20 cycles (depending on the pendulum's initial energy and the progress of the crack) a camera set on a microscope was used to take pictures of the crack.

\section{Experimental results}

In this section, the effects of the impact energy on fatigue life and the evolution of force and contact time during the loading history are presented. Damage evolution and the crack growth process are then analysed.

\subsection{Characterisation of the impact fatigue process}

The number of cycles (impacts) to failure, $N_{\mathrm{f}}$, depends on the level of impact energy, which here refers to the potential energy of the hammer minus losses determined from testing without a sample. Obviously, the lower the energy, the longer the life of specimens and Fig. 2 shows that the relationship between the logarithm of $N_{\mathrm{f}}$ and impact energy is reasonably well presented by a straight line. 


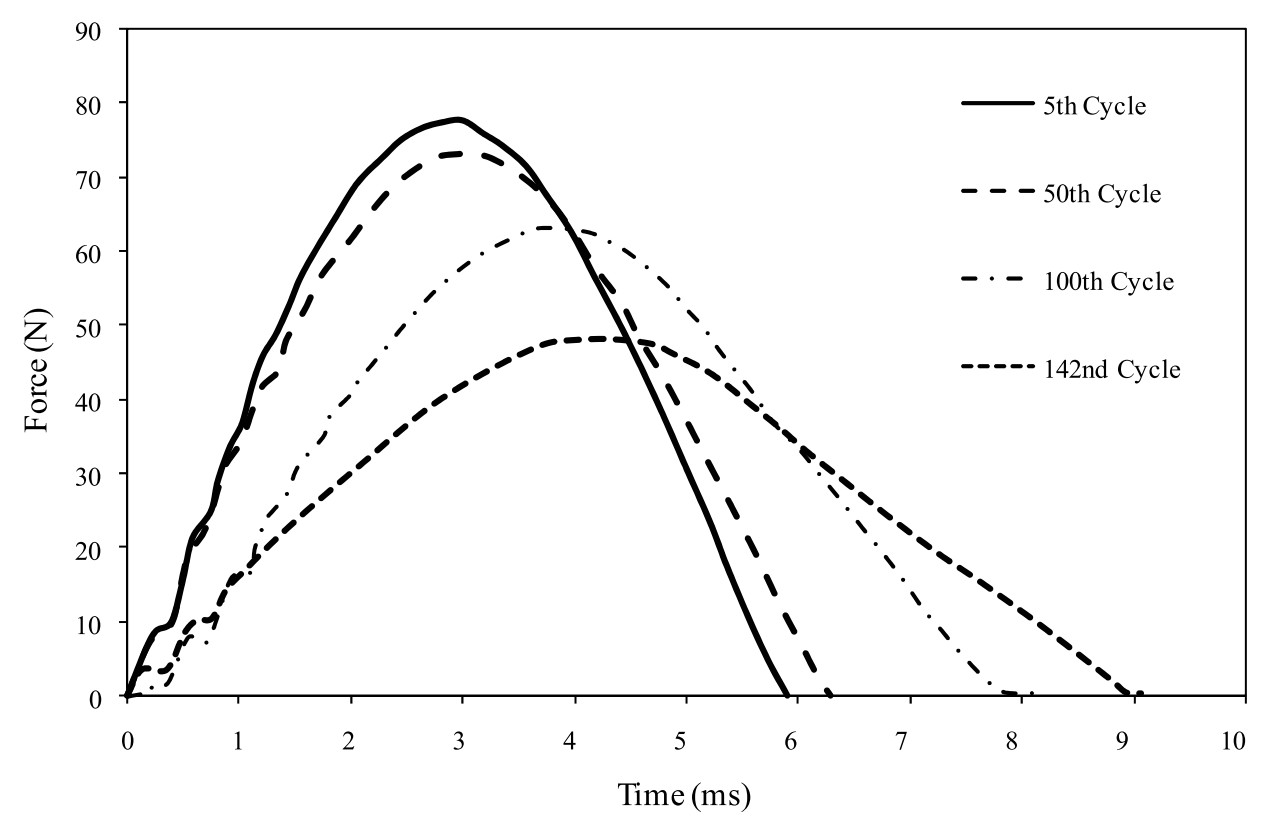

Fig. 3. Effect of repeated impacts at a constant impact energy on the force-time response.

In standard fatigue it is more usual to look for relationships between stress, or force, and $N_{\mathrm{f}}$, than energy, however, in this case, although the impact energy remains constant throughout the test, the maximum force, and hence, stress, does not. This is illustrated in Fig. 3, which shows the force-time plot for impacts at different stages in the impact fatigue life of a sample. It can be seen that the force-time profile changes with the loading history. The force amplitude decreases while the contact time increases. This is due to a change in the dynamic interaction between the hammer and the specimen with increasing damage in the specimen. Damage and cracking results in a decrease in the stiffness of the specimen, these changes becoming more pronounced in the final stages of the specimen's life. This is clearly illustrated in Fig. 4. Figure 4(a) shows the change in the maximum force, $F_{\max }$, normalised with respect to the average maximum force from the first $20 \%$ of impacts, $\mathrm{F}_{\mathrm{avg}}$, with number of fatigue cycles. This normalising parameter was selected rather than the maximum force from the first impact because scatter was sometimes seen in the maximum force from the first few impacts due to small misalignments between the hammer and impact block. The system appeared to self-align after a few impacts and scatter reduced accordingly. In this case the average of the first $20 \%$ of impacts provided a reasonable approximation of the conditions at the early stages of the test whilst reducing scatter, however, with other samples a different normalising parameter may be more appropriate. It can be seen in Fig. 4 that there is little decrease in $\mathrm{F}_{\max }$ for the first 10-20\% of the fatigue life, after which there is a steady decrease, followed by a rapid decrease in the last 10-20\% of the fatigue life. Interestingly, it can be seen that when the number of cycles, $\mathrm{n}$, is normalised with respect to the fatigue life, $\mathrm{N}_{f}$, that almost identical behaviour is seen, regardless of the impact energy. Similar trends can be seen in the case of contact time, as seen in Fig. 4(b).

In Fig. 5, the normalised maximum load is plotted against the normalised contact time. Fig. 5(a) shows that there is a non linear relationship between these two parameters that appears to be independent of the impact energy. In Fig. 5(b) all the data points, regardless of impact energy are plotted on a log-log scale. It can be seen that all the data lies reasonably well on a single straight line, albeit with some scatter, particularly at low contact times. This provides good evidence that the dependence of the maximum force on the contact time can be approximated by the energy independent power law given below:

$$
\frac{F_{\max }}{F_{\mathrm{avg}}}=A\left(\frac{t}{t_{\mathrm{avg}}}\right)^{n}
$$

where $A$ and $n$ are constants, which in this case are 0.98 and -0.91 , respectively. 


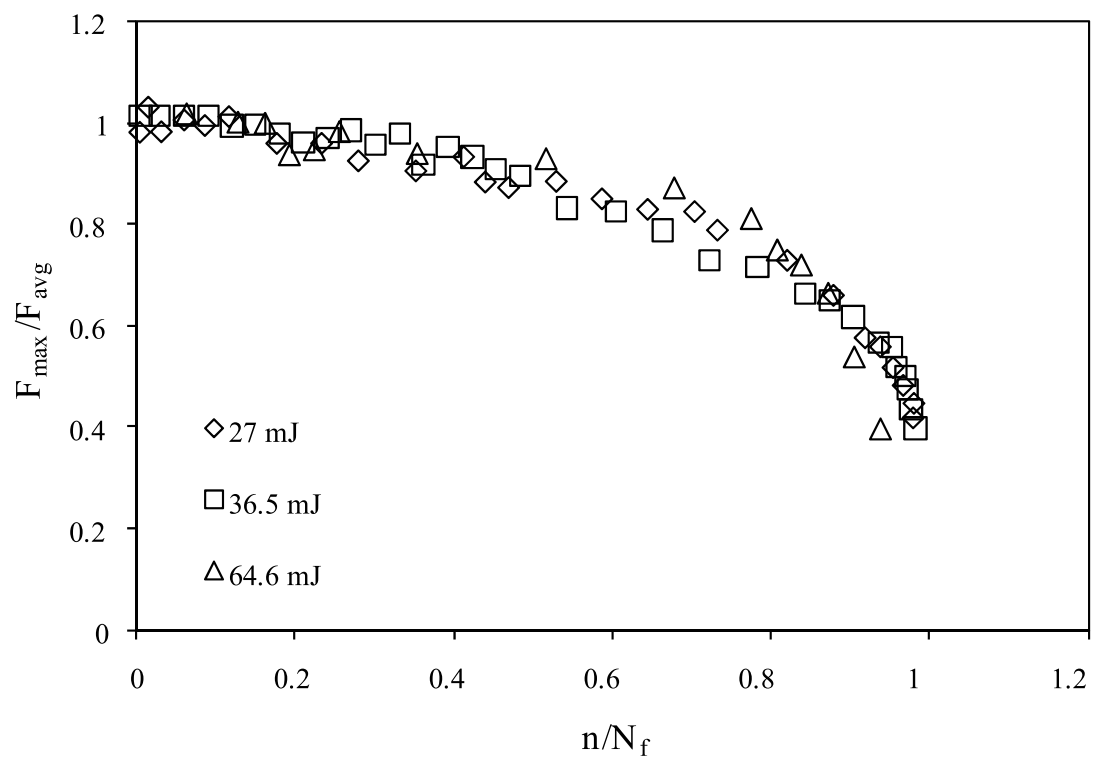

(a)

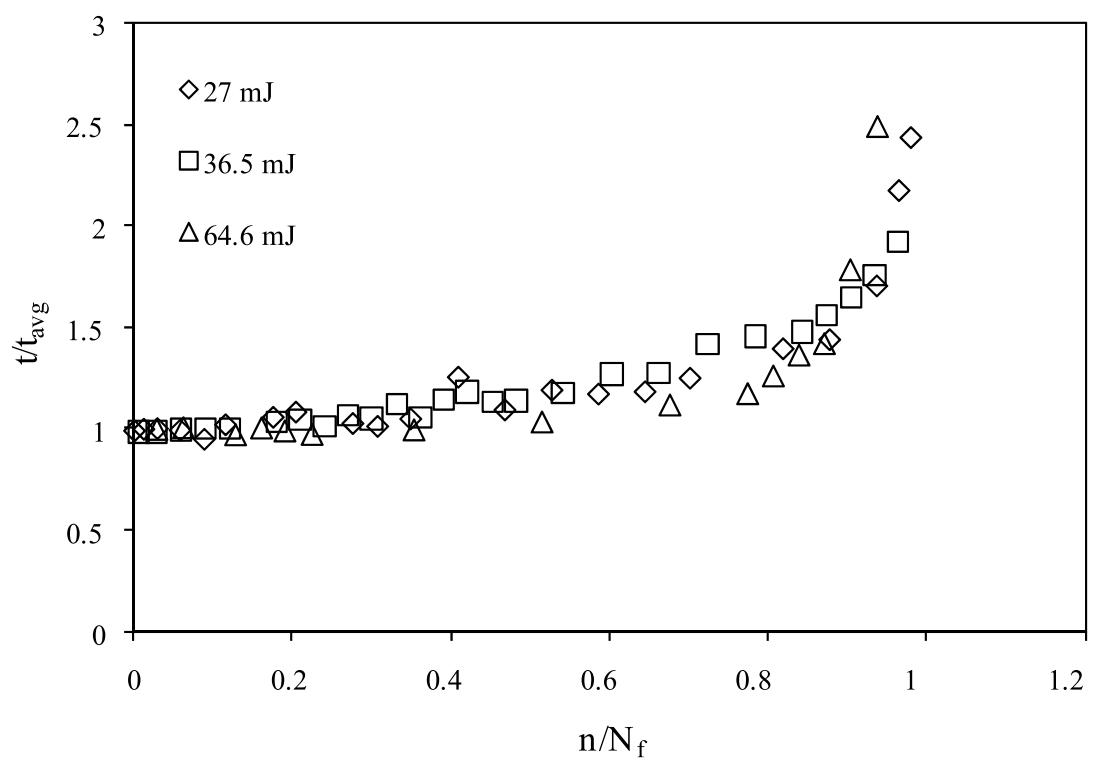

(b)

Fig. 4. Effect of normalised number of cycles $\left(n / N_{f}\right)$ and impact energy on (a) normalised maximum force $\left(F_{\max } / F_{a v g}\right)$ and $(b)$ normalised contact time $\left(\mathrm{t} / \mathrm{t}_{\mathrm{avg}}\right)$.

\subsection{Damage and crack extension}

After studying the general response of the specimen in terms of the force and contact time as a function of repeated impact, it is important to analyse the evolution of damage and propagation of cracks in the sample. From optical observation of the crack development during the impact fatigue testing, it was seen that three main phases existed in the evolution of damage. In the first phase, stress whitening developed at the root of the notch, as seen in Fig. 6(a). This is indicative of micro-damage within the adhesive sample. In the second phase a macro-crack formed at the notch root and began to progress steadily through the sample. This is represented by the region of straight crack 


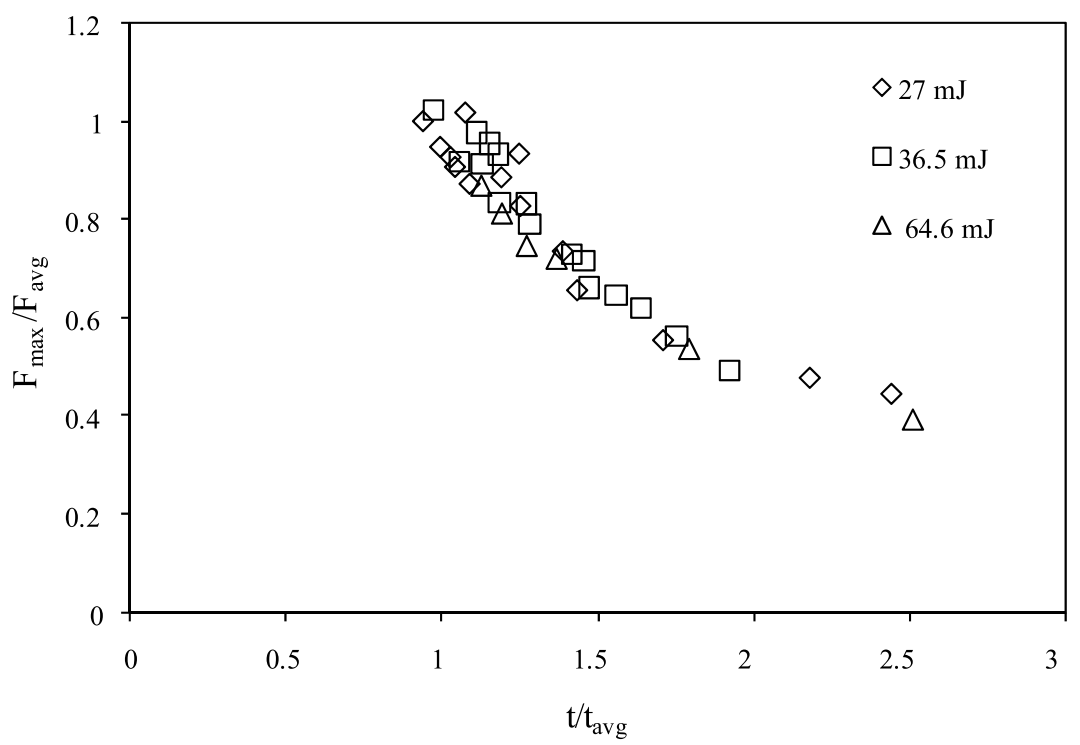

(a)

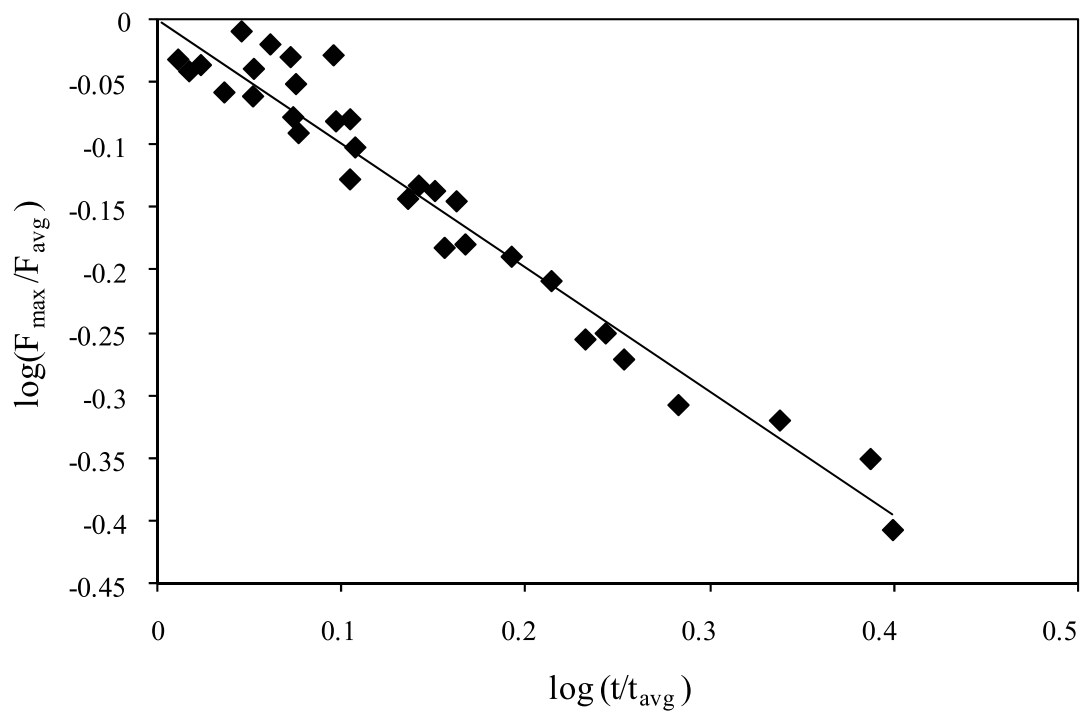

(b)

Fig. 5. Normalised load-time plots on (a) linear and (b) logarithmic axes.

growth in Fig. 6(b). In the third phase, crack propagation accelerated towards complete sample failure. This is characterised by the more convoluted crack path in the bottom half of the sample in Fig. 6(b). In this region there appears to be more damage in the area of the crack.

The three phases described above can be clearly seen in the plot of crack length against normalised number of cycles shown in Fig. 7. It can be seen that for the first $15-25 \%$ of the fatigue life there is no observable crack growth. This corresponds to a damage initiation period, characterised by the stress whitening at the notch root seen in Fig. 6(a). There follows a period of stable crack growth in which the rate of crack growth is approximately constant. In the final $20 \%$ of the fatigue life, there is an acceleration of the crack growth, terminating in complete failure. It can also be seen in Fig. 7 that the proportion of the impact fatigue life spent in each of the phases described above appears to be largely energy independent. A commonality can now be seen in Figs 4, 6 and 7 relating to the three phases of damage evolution discussed above. 


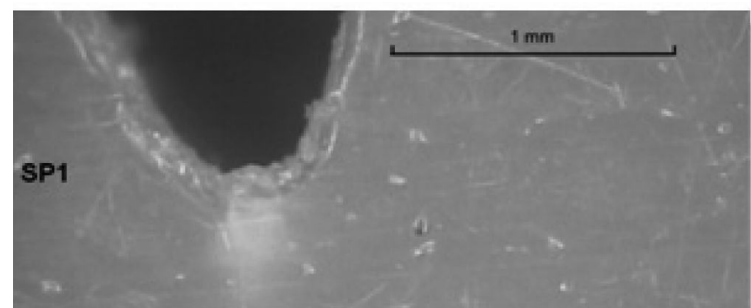

(a)

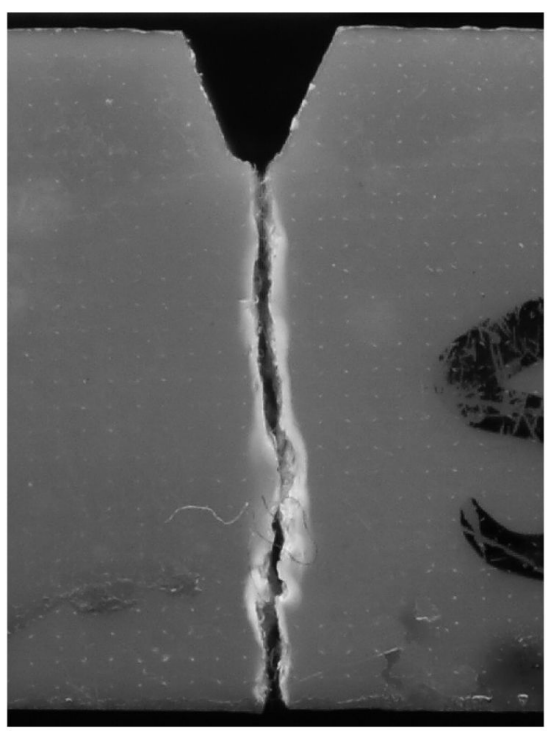

(b)

Fig. 6. (a) Stress whitening at root of notch prior to cracking (b) final crack.

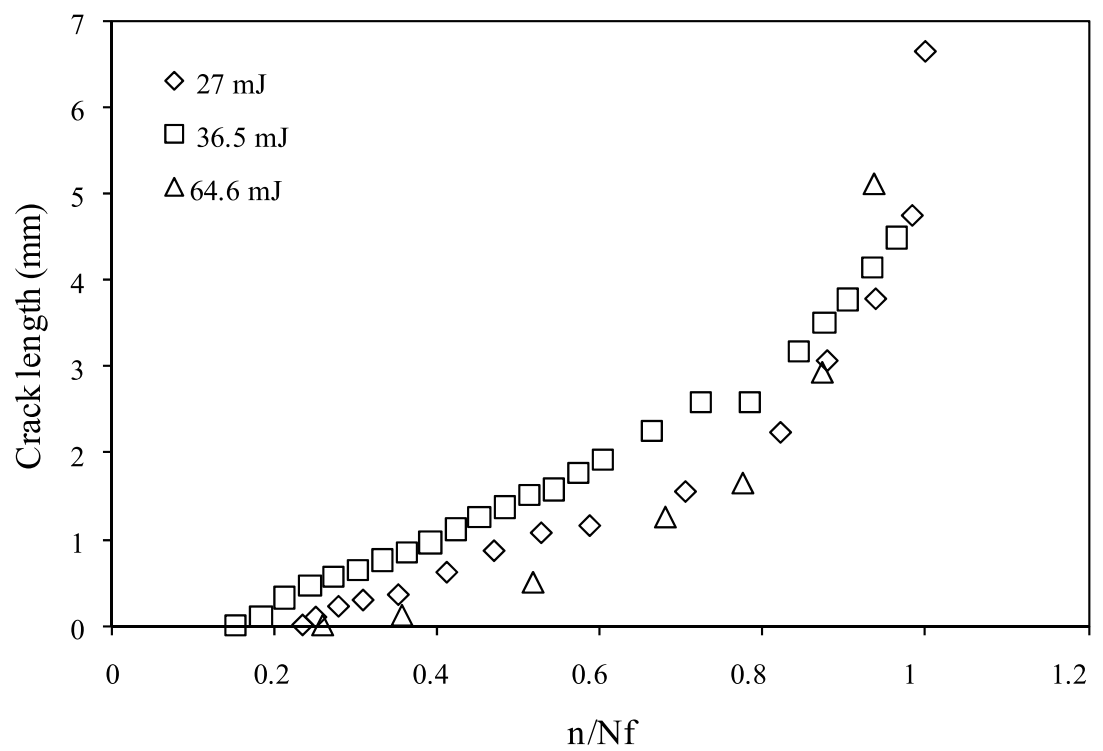

Fig. 7. Crack length as a function of normalised impact fatigue cycles. 


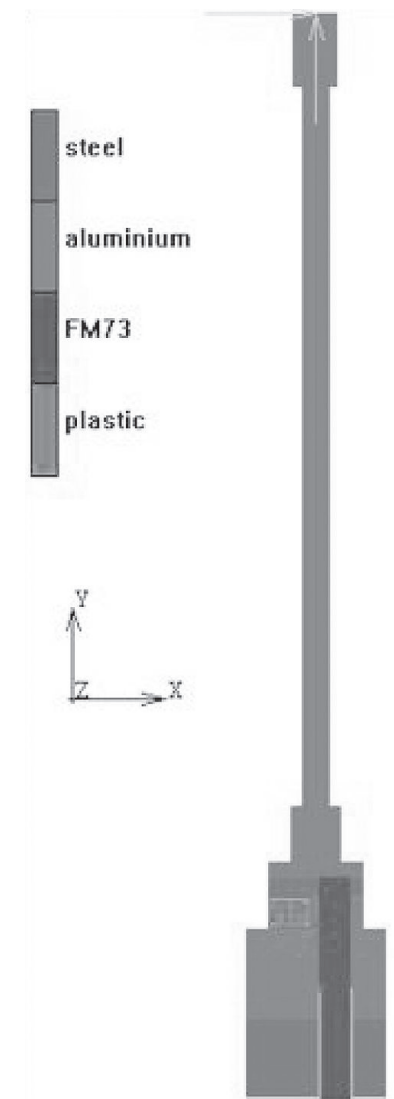

(a)

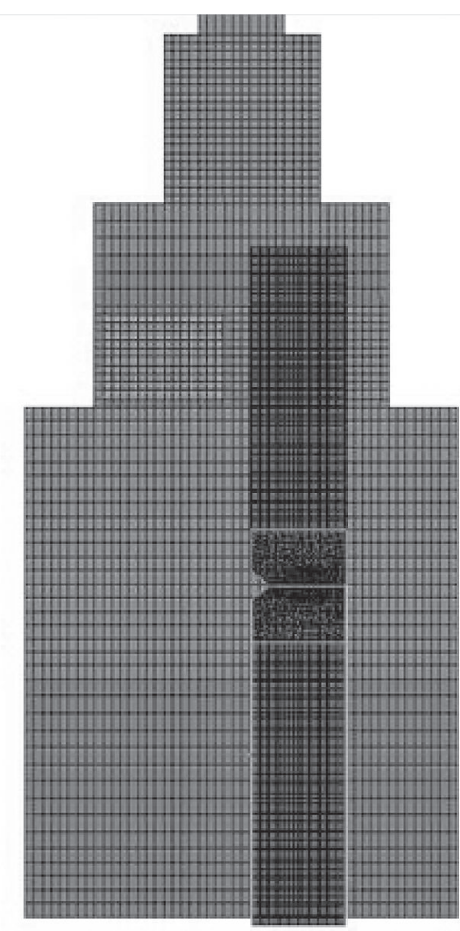

(b)

Fig. 8. Finite element modelling. (a) whole model. (b) Magnification of sample and striking area of hammer.

\section{Development of an impact fatigue crack growth law}

In the preceding section, the impact fatigue behaviour of the epoxy was characterised using various parameters. The next step in the analysis is to investigate methods of predicting the observed behaviour. A common method of predicting fatigue crack growth (FCG) in adhesives is through fracture mechanics, whereby a suitable fracture mechanics parameter is related to the crack growth rate by an empirical crack growth law. In the case of adhesive joints, the crack growth law is often determined from logarithmic plots of the FCG rate, da/dn, as a function of the strain energy release rate range, $\Delta \mathrm{G}$, or maximum strain energy release rate, $\mathrm{G}_{\max }$ (e.g. [1,2]). In order to apply a similar method to the present case we first need to determine the experimental fatigue crack growth rate as a function of crack length. This was achieved by applying an incremental polynomial curve fitting method to the experimental data for crack length against impact cycles and then calculating the derivative to determine the FCG rate as a function of crack length. The next step is to determine a suitable fracture mechanics parameter and $\mathrm{G}_{\max }$ was selected in order to enable comparison with the previously cited work on the standard fatigue of bonded joints. The strain energy release rate was determined from a dynamic finite element analysis (FEA) and by a simple analytical model. In the following sub-sections the numerical model will first be described and validated before moving on to the determination of $\mathrm{G}$ and finally the development of the impact fatigue crack growth model.

\subsection{Finite element model}

The impact test was simulated using a two dimensional, geometrically nonlinear, implicit dynamic finite-element analysis using the commercial FEA software MARC/MENTAT. The transient analysis was implemented using the 


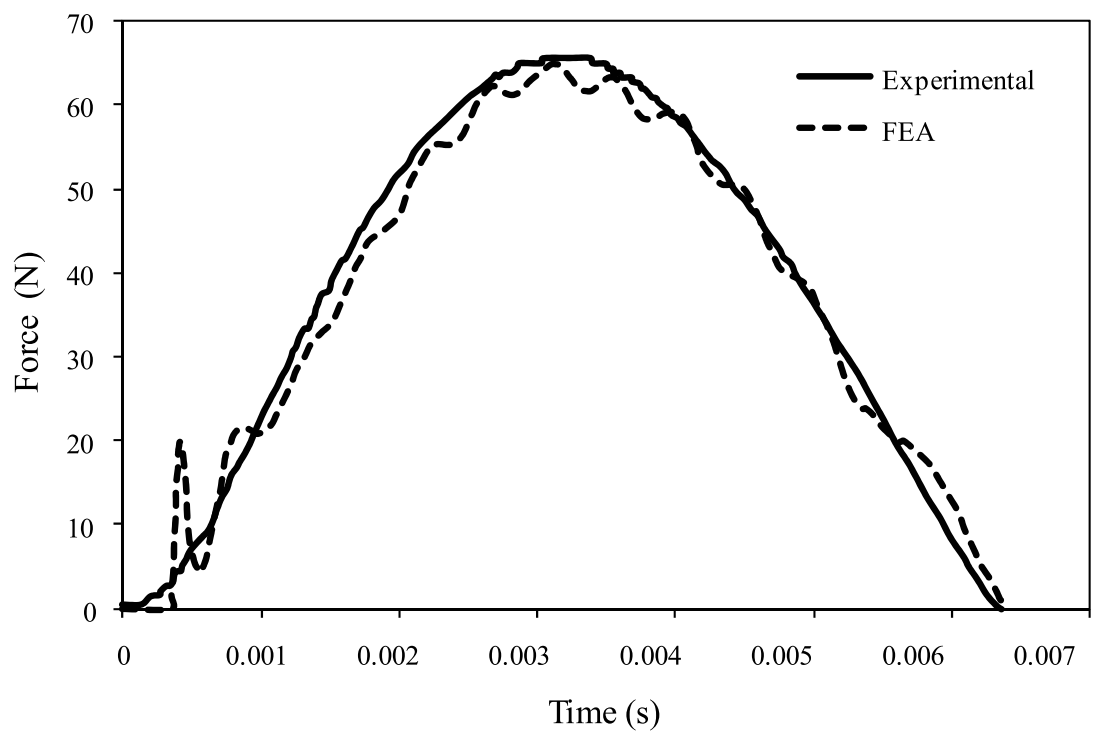

(a)

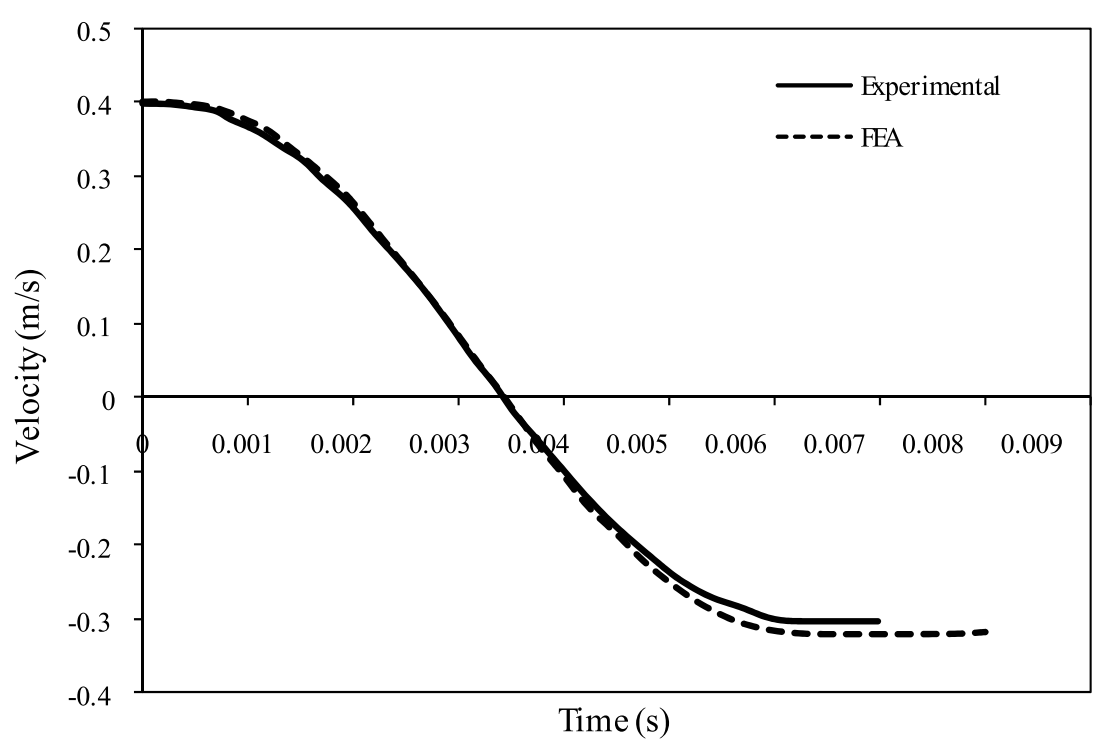

(b)

Fig. 9. Comparison of experimental and computed force (a) and velocity (b) as functions of time.

single step Houbolt integration method recommended for dynamic implicit contact analyses. Quadratic elements with mid sided nodes and formulated using the full integration method were used in the finite element mesh. Material properties were obtained from high strain rate quasi-static tests and linear material properties were assumed in the analyses. A model of the hammer was defined to represent the geometry of the real hammer used in the testing, ensuring that the weight distribution was the same as in the real hammer. The boundary and initial conditions were defined according to those in the experiments. To simulate the centre of rotation of the hammer, a node at its upper extremity was fixed in the $\mathrm{x}$ and $\mathrm{y}$ directions, leaving it unconstrained only in the rotational degree of freedom. The hammer was placed at a short distance from the specimen and a prescribed velocity applied to it. Figure 8 shows the finite element model used. The initial FE model was developed with the edges of the lower part of the specimen constrained in the $\mathrm{x}$ and $\mathrm{y}$ directions to simulate the effect of the fixture. However, when compared with the experimental data, the calculated magnitudes of force were too high and the contact time too small. A stiff 


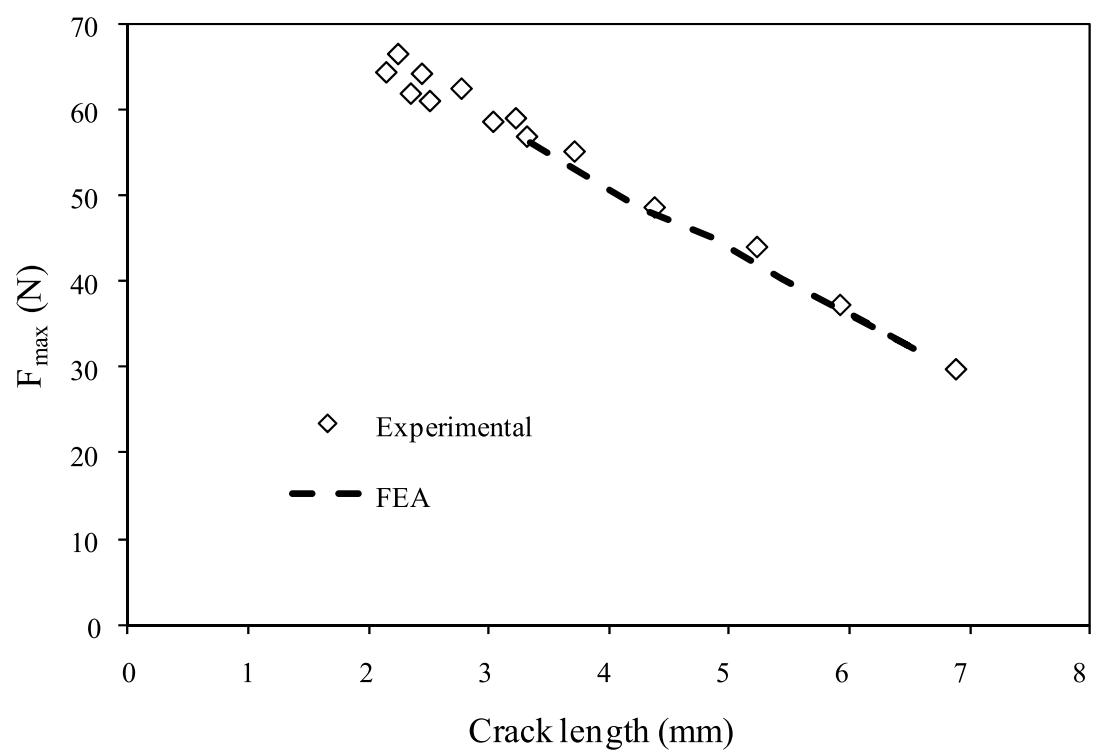

(a)

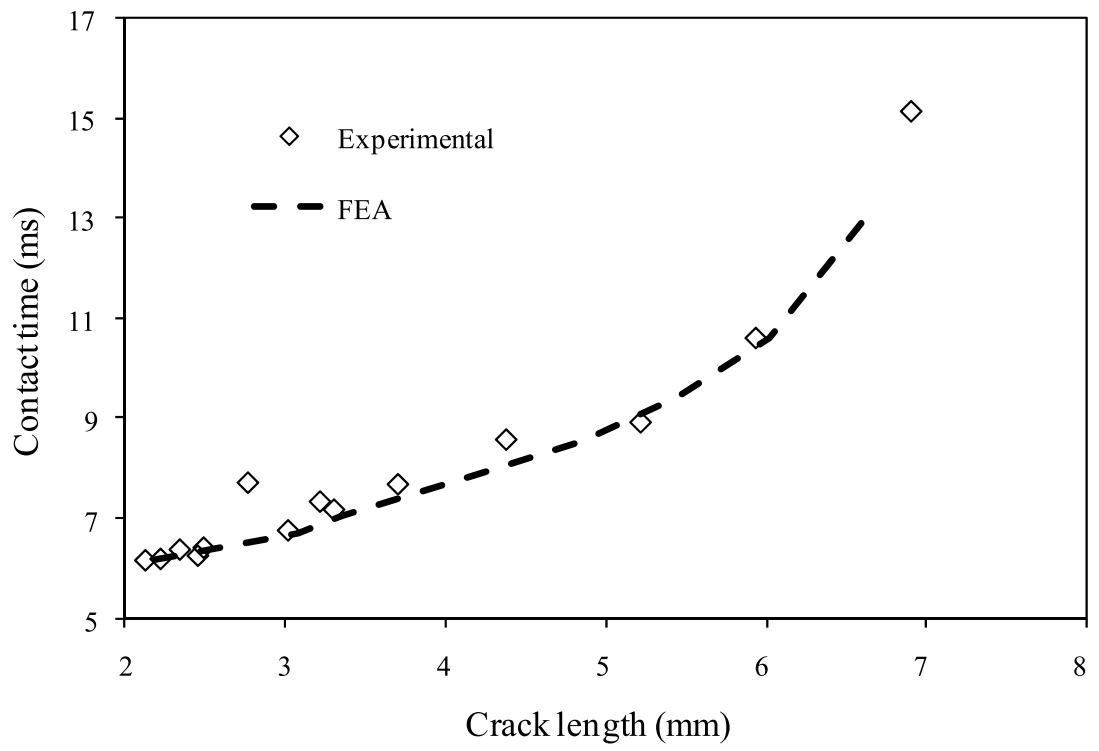

(b)

Fig. 10. Comparison of experimental and computed maximum force (a) and contact time (b) as functions of crack length.

spring coupling was then introduced between the sample and a fixed base to represent the finite stiffness of the real fixture conditions. This increased energy dissipation, decreasing the force and increasing the contact time. The spring stiffness was adjusted to provide the best fit with the experimental results. A comparison of the force-time plot predicted using this model with the experimental results can be seen in Fig. 9(a) It can be seen that both contact time and maximum force agree well with the experimental data. Further validation was obtained by comparing the predicted change in velocity of the hammer with time, as shown in Fig. 9(b). It can be seen that the velocity decreases rapidly as it strikes the sample and then accelerates in the opposite direction, as expected. The figure also shows that there is excellent agreement between the predicted and measured velocity data.

The finite element model was then used to predict the change in maximum force and contact time as cracks of 
increasing size were introduced to the sample. The results are compared with the experimental values in Fig. 10 and, again, it can be seen that there is excellent agreement between the FEA predicted and experimental measured data. It is also interesting to see that when the maximum force is plotted as a function of crack length, rather than cycles, all the data fits reasonably well to a straight line. These results provide confidence in the FEA model, which is used to determine the strain energy release rate in the next section.

\subsection{Determination of strain energy release rate}

In order to obtain the strain energy release rate using the dynamic finite element model described above, the crack propagation process was simulated by uncoupling nodes along the crack propagation line and re-meshing after each increment of crack growth. The strain energy release rate was then calculated using the subroutine for the determination of the J-integral in Marc. The maximum value of the strain energy release rate, $\mathrm{G}_{\max }$, was determined at the point of maximum force in the dynamic load-time response of the FEA model.

Determination of $\mathrm{G}_{\max }$ from analytical expressions was also investigated as this is a simpler and quicker method than using dynamic FEA. For the notched cantilever the stress intensity factor can be presented using the following form [11]:

$$
K_{\mathrm{I}}=\frac{6 M\left(0.38+1.3 \frac{a}{w}-1.2 \frac{a^{2}}{w^{2}}\right)}{b \sqrt{\pi}(w-a)^{3 / 2}}
$$

where $M$ is the bending moment, $a$ is the notch size, $b$ is sample thickness and wis the sample width. A dynamic formulation of Eq. (5) can be derived using the method proposed by Fengchun et al. [8] to calculate the strain energy release rate in dynamic conditions. The equation of motion for a spring-mass system characterised by mass $m_{\mathrm{e}}$ and displacement $u(t)$ is:

$$
m_{\mathrm{e}} \ddot{u}(t)+K(a) u(t)=F(t)
$$

where $F(t)$ is the impact load and $K(a)$ is the stiffness of the specimen.

A solution of this equation with initial conditions $u(0)=\dot{u}(0)=0$ is:

$$
u(t)=\frac{I(t)}{\omega_{1} m_{\mathrm{e}}}
$$

where:

$$
I(t)=\int_{0}^{t} F(\tau) \sin \left(\omega_{1}(t-\tau)\right) d \tau
$$

and $\omega_{1}$ is the natural frequency of the system, which is given by:

$$
\omega_{1}=\sqrt{\frac{K(a)}{m_{\mathrm{e}}}}
$$

At maximum force the force is the product of the stiffness and displacement. Substituting for stiffness and displacement from Eqs (7) and (8) gives:

$$
F_{\max }=\omega_{1} I(t)
$$

In our impact fatigue tests, the maximum moment is equal to the maximum measured force multiplied by a lever of $22 \mathrm{~mm}$. Then the stress intensity factor for dynamic conditions is:

$$
K_{\mathrm{I}}=\frac{0.132 \omega_{1} I(t)\left(0.38+1.3 \frac{a}{w}-1.2 \frac{a^{2}}{w^{2}}\right)}{b \sqrt{\pi}(w-a)^{3 / 2}}
$$

Once the dynamic stress intensity factor is known, the dynamic strain energy release rate is calculated using the following formula for plane strain conditions: 


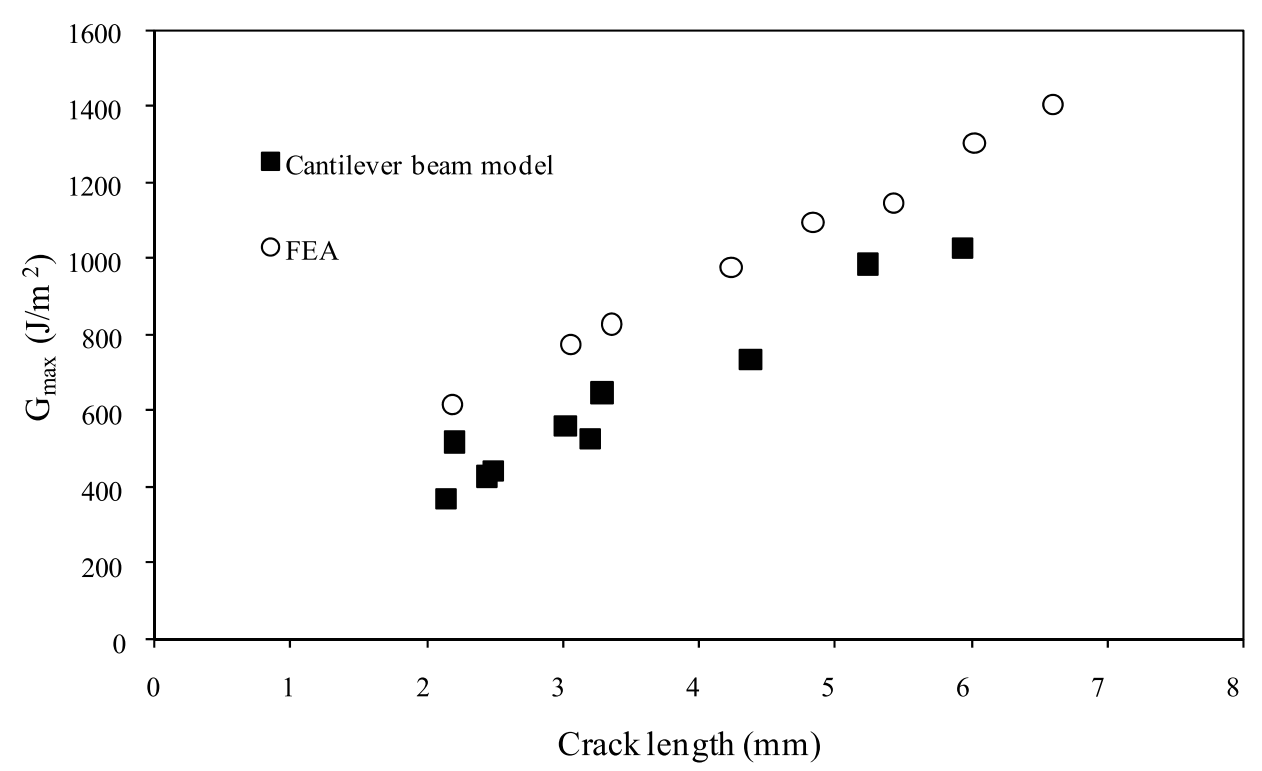

Fig. 11. Maximum dynamic strain energy release rate as a function of crack length.

$$
G_{\mathrm{C}}=\frac{K_{\mathrm{C}}\left(1-\nu^{2}\right)}{E}
$$

where $E$ is the Young's modulus and $\nu$ is the Poisson's coefficient of the material.

A comparison of the calculated maximum dynamic strain energy release rate as a function of crack length is shown in Fig. 11. It can be seen that although the simple analytical model underestimates the level of the strain energy release rate, the slopes of the two relationships are close. Both the analytical and FEA models indicate an approximately linear increase in $\mathrm{G}_{\max }$ with crack length.

\subsection{Impact fatigue crack growth law}

In the preceding sections, the methods of determining the impact fatigue crack growth rate, da/dn, and maximum dynamic strain energy release rate, $\mathrm{G}_{\max }$, were described. The next step is to investigate the relationship between these two parameters in order to devise an impact fatigue crack growth model. Paris et al. [9] proposed a power law relationship between the FCG rate for metals in fatigue with the stress intensity factor range. A similar relationship is proposed here for impact fatigue, namely:

$$
\frac{d a}{d n}=C G_{\max }^{n}
$$

This relationship is explored in Fig. 12 by plotting $\mathrm{G}_{\max }$ against da/dn on logarithmic axes. It can be seen in the figure that both the analytical and FEA methods of determining $G_{\max }$ result in a linear relationship between the logarithms of da/dn and $\mathrm{G}_{\max }$, thus confirming the applicability of Eq. (12) to the case of impact fatigue in the epoxy adhesive tested. Once the crack growth law is established, FEA based numerical integration techniques can be used to predict the impact fatigue crack growth in samples of different geometry, e.g. by using the procedures described in Abdel Wahab et al. [1].

\section{Summary and conclusions}

This paper presents a detailed study of crack generation and growth in notched specimens of FM73 adhesive exposed to repeated low-energy impacts. Three main phases were observed in the evolution of damage and cracking in the samples under repeated impacts. In the first phase, which occupies some $20 \%$ of the specimen's life, there is 


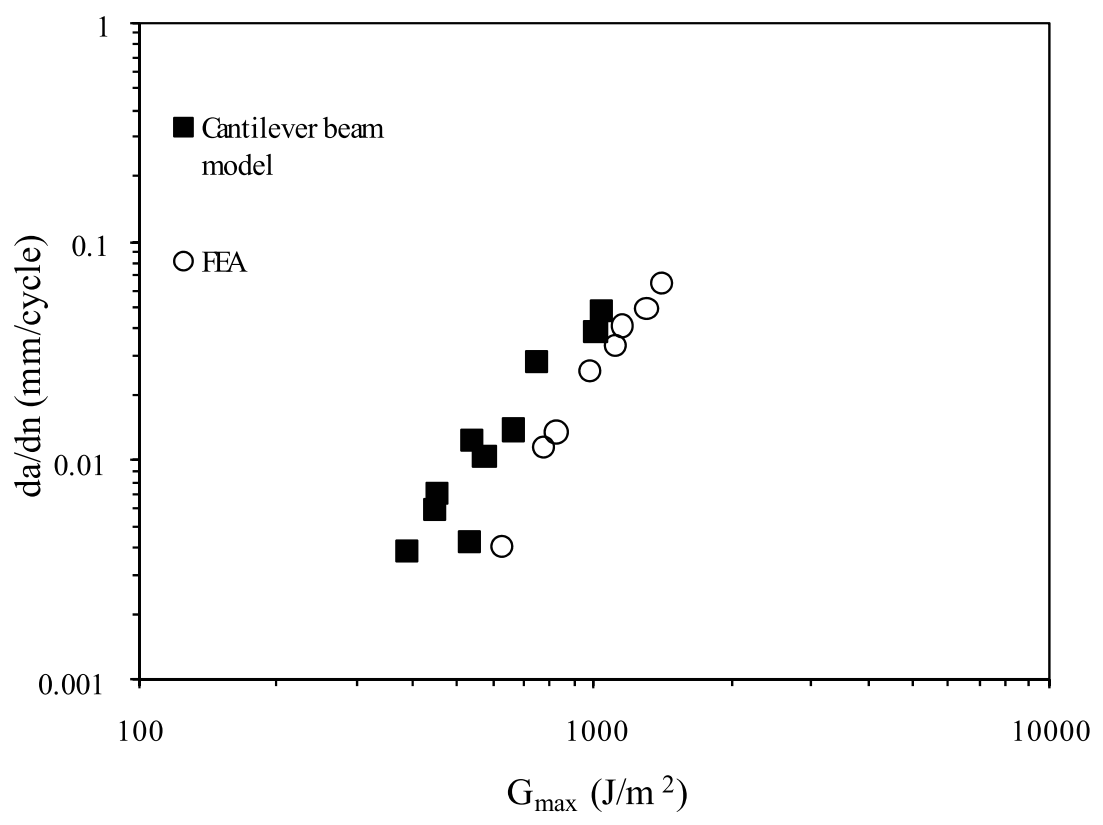

Fig. 12. Impact fatigue crack growth plot.

damage initiation but no macro-cracking of the sample. In this phase there is little change in the maximum force and contact time. In the second phase, which lasts for a significant part of the life in service, there is an approximately linear increase in crack length, accompanied by approximately linear decrease in maximum force and increase in contact time. In the final phase, crack growth accelerates, with accompanying increases in the rates of change in maximum force and contact time. It was seen that plots of maximum force, contact time and crack length as functions of the number of cycles normalised by the fatigue life were independent of the applied impact energy, thus enabling effects over a range of energies to be approximated from tests at a single energy. However, in order to use this relationship, the applicable range needs to be determined. It is envisaged that at very high or low impact energies deviations from this general trend may occur.

In the second part of this paper a crack growth law for the impact fatigue of the epoxy adhesive material tested was developed. It was seen that a Paris-type power law relationship between the crack growth rate and the maximum dynamic strain energy release rate could be used to describe the crack growth behaviour. Although this relationship has only been proven for a single material, it is likely to be applicable to other materials and the experimental and analysis methods described in this paper can be used to define the crack growth law constants for any material. It is commonly seen in standard fatigue that the Paris-type relationship only holds over a limited range. At high strain energy release rates crack growth accelerates and at low strain energy release rates there is often a threshold values, below which crack growth is negligible. Although these regions weren't seen within the range of impact energies investigated in this work, users should be careful to only use the proposed crack growth law over the range it has been validated as extrapolation beyond this range can lead to potentially large errors.

\section{Acknowledgement}

The authors are very grateful for a partial financial support by the Royal Society within the framework of its International Joint Projects scheme.

\section{References}

[1] M.M. Abdel Wahab, I.A. Ashcroft, A.D. Crocombe and P.A. Smith, Numerical prediction of fatigue crack propagation lifetime in adhesively bonded structures, Int J Fatigue 24 (2001), 705-709. 
[2] M.M. Abdel Wahab, I.A. Ashcroft, A.D. Crocombe and P.A. Smith, Finite element prediction of fatigue crack propagation lifetime in composite bonded joints, Composites Part A 35 (2004), 213-222.

[3] I.A. Ashcroft, J.P. Casas-Rodriguez and V.V. Silberschmidt, Mixed-mode crack growth in bonded composite joints under standard and impact-fatigue loading, J Mater Sci 43 (2008), 6704-6713.

[4] J.P. Casas-Rodriguez, I.A. Ashcroft and V.V. Silberschmidt, Damage evolution in adhesive joints subjected to impact fatigue, $J$ Sound Vibrat 308 (2007), 467-478.

[5] J.P. Casas-Rodriguez, I.A. Ashcroft and V.V. Silberschmidt, Delamination in adhesively bonded CFRP joints: Standard fatigue, impactfatigue and intermittent impact, Compos Sci Technol 68 (2008), 2401-2409.

[6] J.P. Casas-Rodriguez, I.A. Ashcroft and V.V. Silberschmidt, Damage in adhesively bonded CFRP joints: Sinusoidal and impact-fatigue, Compos Sci Technol 68 (2008), 2663-2670.

[7] B. Chen, D.A. Dillard, J.G. Dillard and R.L. Clark, Crack path selection in adhesively bonded joints: the roles of external loads and specimen geometry International, J Fracture 114 (2002), 167-190.

[8] J. Fengchun, L. Ruitang, Z. Xiaoxina, K.S. Vecchiob and A. Rohatgi, Evaluation of dynamic fracture toughness $\mathrm{K}_{i d}$ by Hopkinson pressure bar loaded instrumented Charpy impact test, Engng Fracture Mech 71 (2004), 279-287.

[9] O.C. Paris, M.P. Gomez and W.E. Anderson, A rational analytic theory of fatigue life, Tren Eng 13 (1961), 9-14.

[10] A. Oechsner and L.F.M. da Silva, Advances in Modelling Adhesively Bonded Joints, Springer Publishing, 2008.

[11] S.E. Slavin and G.T. Beswick, Instrumented Izod impact testing, J Appl Polymer Sci 49 (1993), 1065-1070.

[12] H. Tada, The Stress Analysis of Cracks Handbook. Del Research Corp., Hellertown, PA, 1973. 

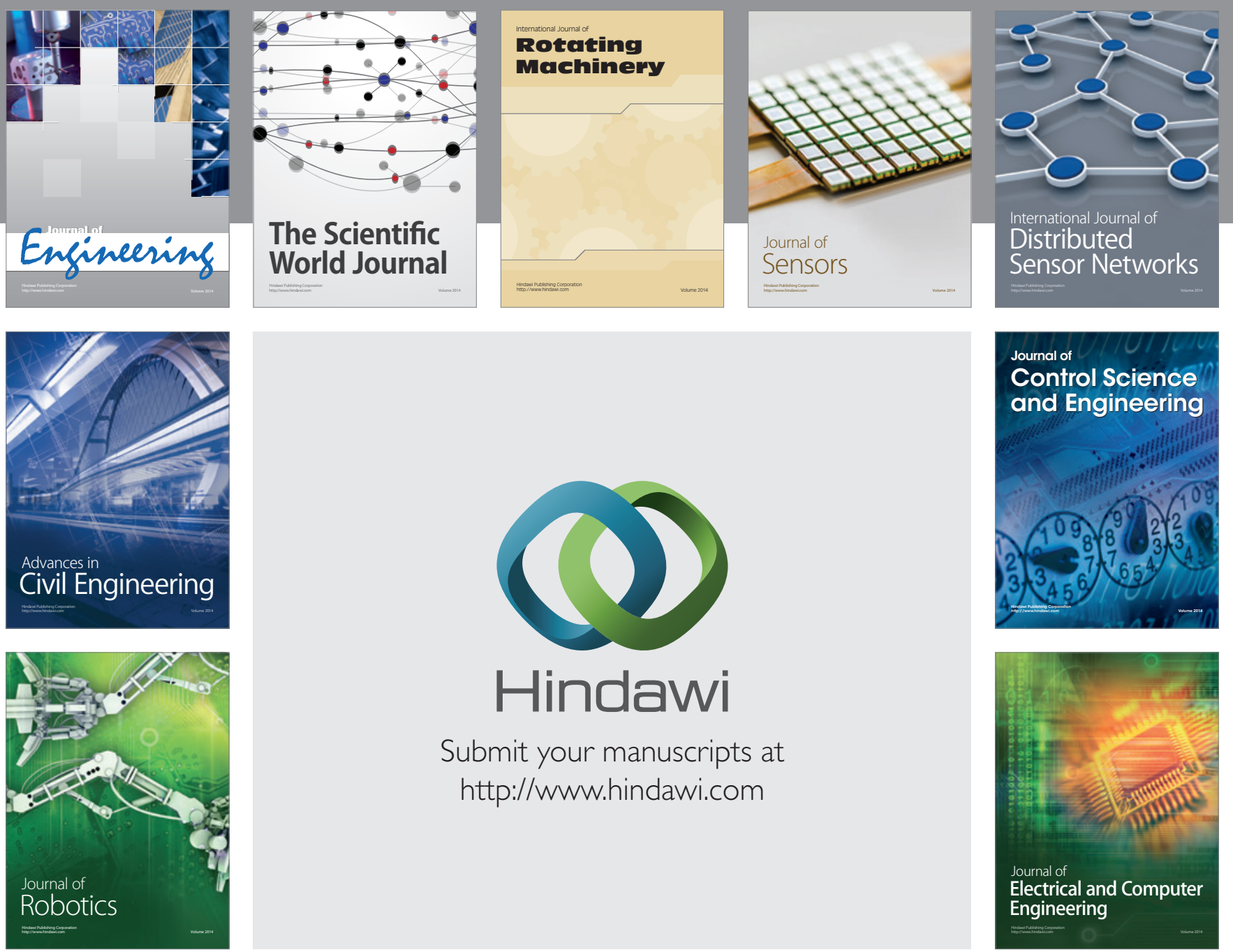

Submit your manuscripts at

http://www.hindawi.com
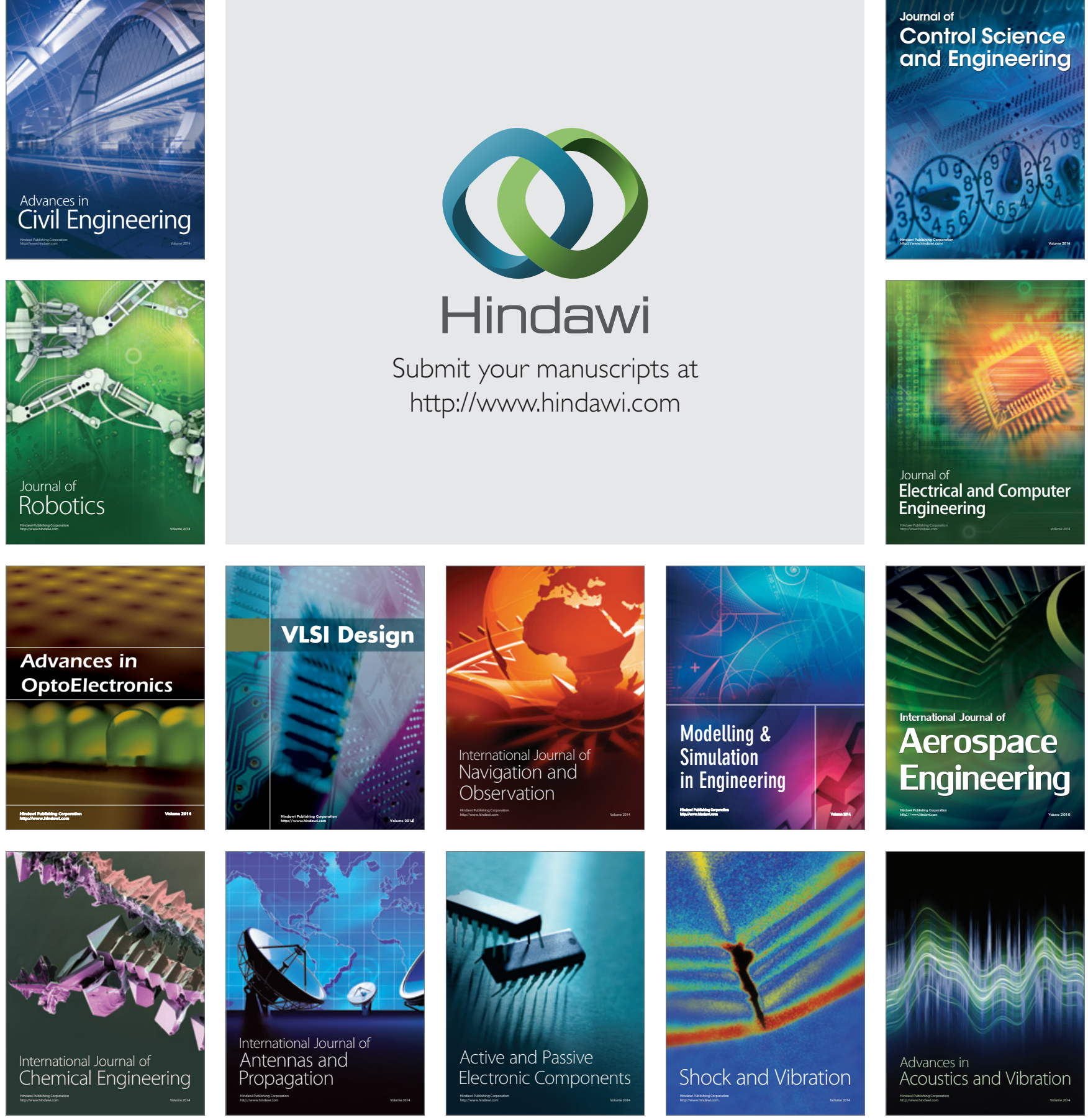